\title{
Ultrasound for Critical Care Physicians: Unchain My Heart
}

\author{
William Mansfield, MD \\ Michel Boivin, MD \\ Division of Pulmonary, Critical Care and Sleep Medicine \\ Department of Medicine, \\ University of New Mexico School of Medicine \\ Albuquerque, NM USA
}

A 46-year-old man presented after a motor vehicle collision. He suffered abdominal injuries (liver laceration, avulsed gall bladder) which were successfully managed nonoperatively. The patient remained intubated on mechanical ventilation and remained hypotensive after the injuries resolved. The patient required norepinephrine at low doses to maintain a normal blood pressure. It was noted the patient had a history of remote tricuspid valve replacement. A bedside echocardiogram was then performed to determine the etiology of the patient's persistent hypotension after hypovolemia had been excluded.

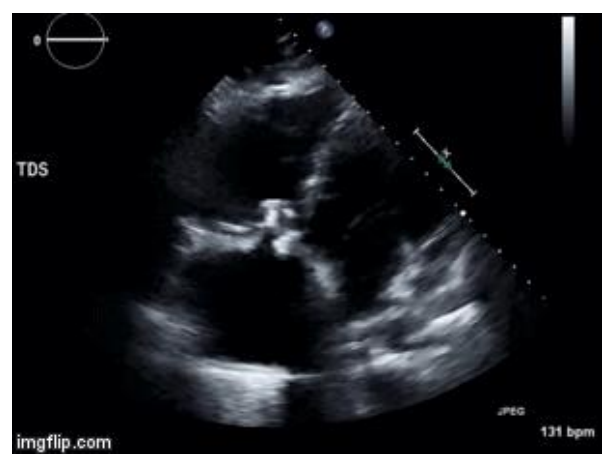

Video 1. Apical four chamber view centered on the right heart.

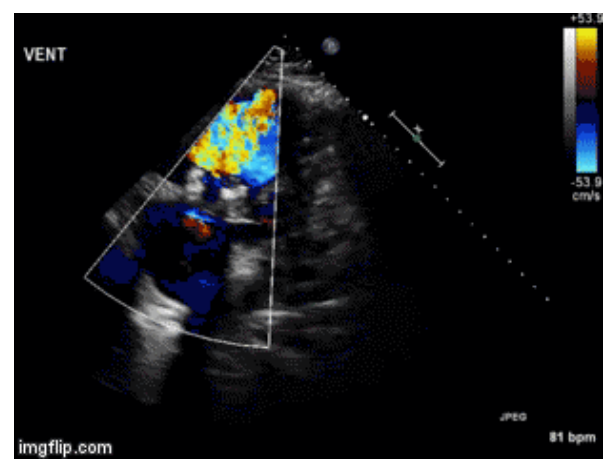

Video 2. Apical four chamber view centered on the right heart, with color Doppler over the right atrium and ventricle. 


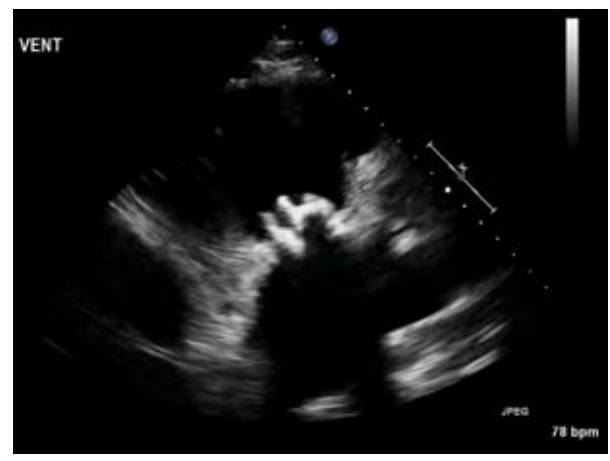

Video 3. Right ventricular inflow view

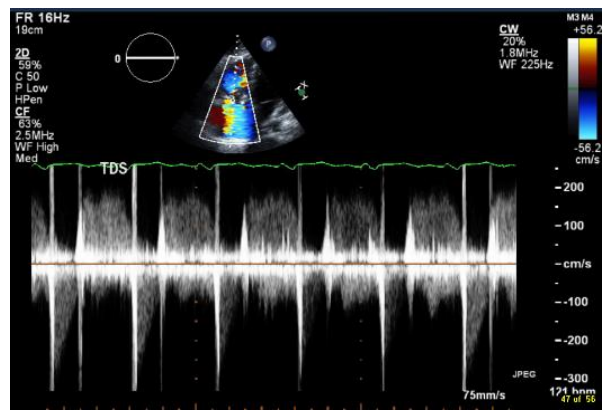

Figure 1. Continuous-wave Doppler tracing through the tricuspid valve.

What tricuspid pathology do the following videos and images demonstrate?

1. Mobile vegetation

2. Tricuspid Regurgitation

3. Tricuspid Stenosis

4. All of the above 


\section{Correct! \\ 4. All of the above}

This case demonstrates a prosthetic tricuspid valve that has developed tricuspid regurgitation, stenosis and has a mobile vegetation. The tricuspid regurgitation is best recognized by the blue jet seen in the right atrium in Figure 2.

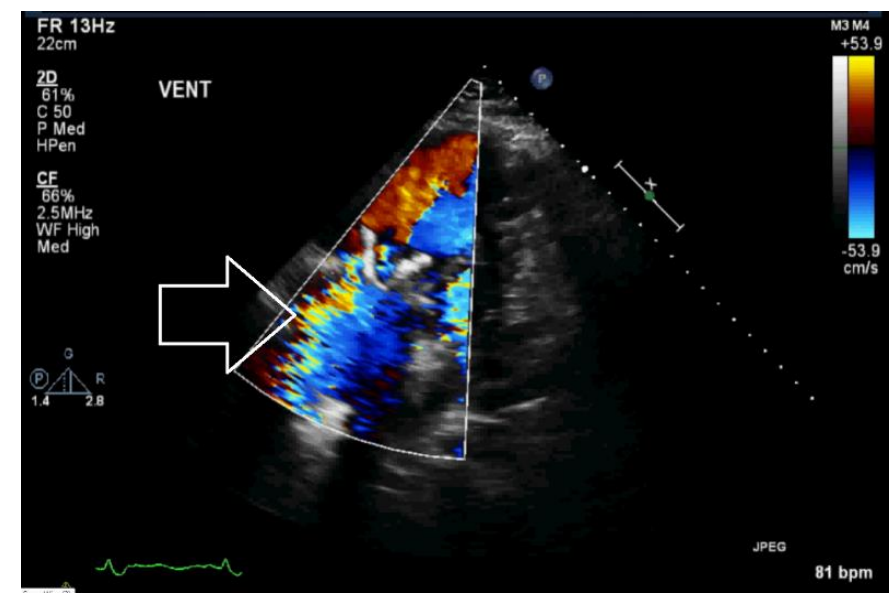

Figure 2. Still image of Video 2, with color Doppler over right atrium in systole. Arrow is pointing to tricuspid regurgitation jet.

Tricuspid stenosis is also seen. This is a much less common lesion. Tricuspid stenosis is demonstrated by the elevated peak gradient in diastole, which is measured at 23 $\mathrm{mmHg}$ (see Figure 3) and indicates significant stenosis (1). Another clue to the tricuspid stenosis is the presence of flow convergence (radiating semi circles on the atrial side of the valve on color Doppler) which are indicative of flow acceleration as the atrial outflow approaches the narrowed tricuspid valve (2). The stenosis of the tricuspid valve cause the flow to accelerate, the velocity to change, and thus the Doppler signal changes color.

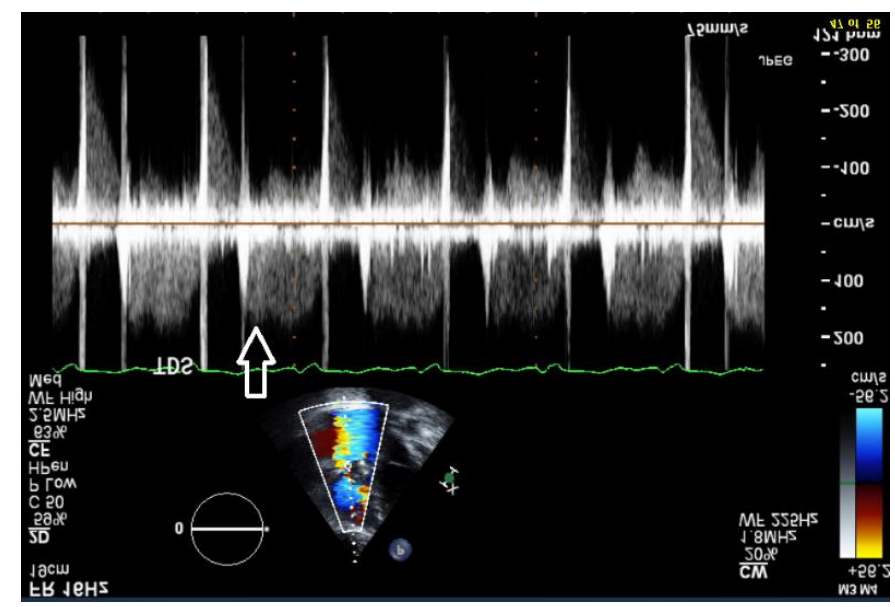


Figure 3. Continuous wave Doppler with arrow pointing to diastolic peak gradient.

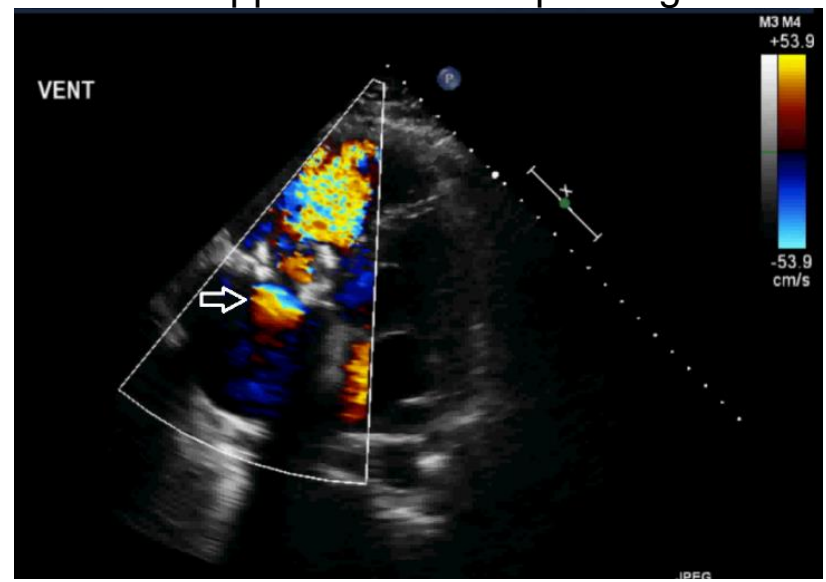

Figure 4. Still image of Video 2, with color Doppler of flow through the tricuspid valve in diastole. Arrow pointing to the zone of flow convergence (semi circles of velocity change on atrial side of tricuspid valve)

Finally, a mobile vegetation is seen on the valve. This is best visualized in the still image demonstrated (below) of the tricuspid valve seen in the right ventricular inflow view. As seen in Video 1 and 3, the vegetation is clearly mobile.

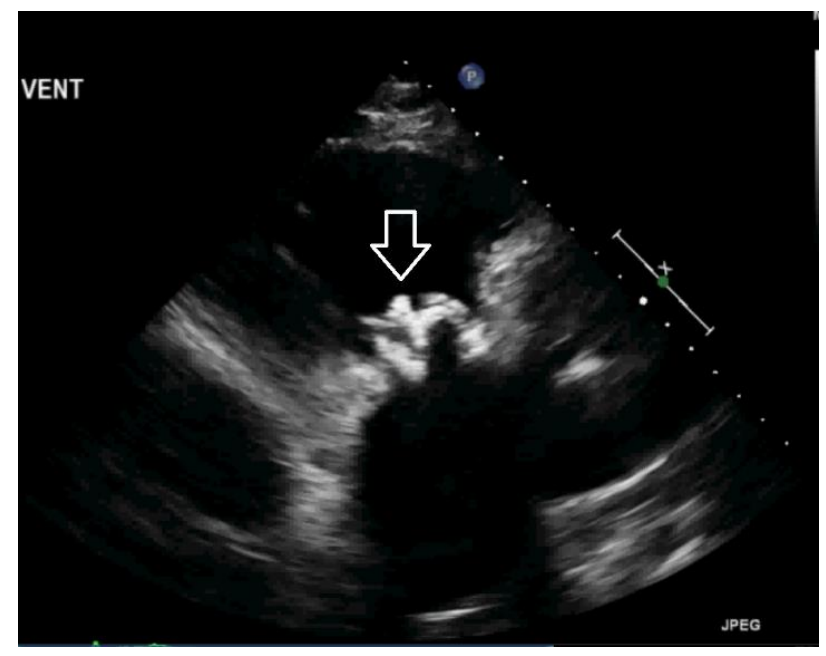

Figure 5. Still image of Video 3. Arrow is pointing to the vegetation.

The patient was managed conservatively and treated with antibiotics over 6 weeks. The patient's volume status was optimized, keeping the patient relatively volume loaded, to maintain preload to the right ventricle. With these treatments, the patient was slowly weaned off vasopressors over a period of two weeks.

Conclusion. This is a complicated echocardiographic case of tricuspid valve vegetation, stenosis and regurgitation all seen in the same patient with a prosthetic tricuspid valve. 


\section{References}

1. Baumgartner H, Hung J, Bermejo J, Chambers JB, Evangelista A, Griffin BP, lung B, Otto CM, Pellikka PA, Qui-ones M; American Society of Echocardiography; European Association of Echocardiography. Echocardiographic assessment of valve stenosis: EAE/ASE recommendations for clinical practice. J Am Soc Echocardiogr. 2009 Jan;22(1):1-23. [CrossRef] [PubMed]

2. Veyrat C, Kalmanson D. Direction, site of origin and duration of jets: implications in the color Doppler assessment of valvar lesions. With emphasis on pure and combined mitral and bicuspid aortic lesions. Int J Card Imaging. 1993 Sep;9(3):15768. [CrossRef] [PubMed] 